\title{
Communication: The key to improving the prostate cancer patient experience
}

by Marian Waldie and Jennifer Smylie

\section{Abstract}

In 2010, an estimated 24,600 Canadian men were diagnosed with prostate cancer (Canadian Cancer Society, 2011). Upon diagnosis, men and their family members begin an arduous journey of information gathering surrounding prostate cancer and its various forms of treatment. Men have to consider the impact a treatment may potentially have on their quality of life and, frequently, they experience decisional conflict and require support.

In May 2008, the Prostate Cancer Assessment Clinic opened to receive men for an evaluation of a possible prostate cancer. Our inter-professional model of care provides support, guidance and education to our patients from assessment to diagnosis and treatment planning. A major goal of our diagnostic assessment unit has been to improve the patient experience.

Communication is defined as "to make known, to exchange information or opinions" (Cayne, Lechner, et al., 1988). Nursing is the critical link for information exchange that is patient-centred and collaborative. The focus of this paper will highlight the development and implementation of nurse-led initiatives within our program to improve the prostate cancer patient experience. These initiatives include: a patient information guide, prostate biopsy care, patient resources, community links, surgery education classes and implementation of a decision aid. Communication is the key.

Men undergoing assessment and diagnosis of prostate cancer face numerous challenges throughout the prostate cancer patient journey. Today, it is estimated that one in seven Canadian men will develop prostate cancer over their lifetime (Canadian Cancer Society, 2011). A diagnosis of prostate cancer may be one of the most stressful events in a man's life. The increasing complexity of care and the variety of treatment choices available including surgery, radiotherapy, active surveillance, watchful waiting and hormonal therapy can be overwhelming for men and their families. They often ask "What is best for me?" Effective communication between the nurse, patient and family, the inter-professional team and community partners is the key to improving the prostate cancer patient experience.

\section{Background}

In 2006, an innovative regional cancer surgery model to improve access to quality cancer surgery and decrease wait times was launched by the Champlain Regional Cancer Program in eastern

\section{About the authors}

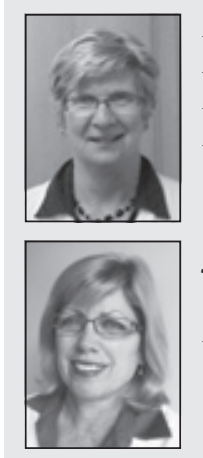

Marian Waldie, $R N, B S c N, C O N(C)$, Oncology Nurse, Cancer Assessment Clinic, The Ottawa Hospital, 501 Smyth Rd., Ottawa, ON K1H 8L6. Email: mwaldie@TOH.on.ca (Preferred author for correspondence)

Jennifer Smylie, RN, BN, MHSM, Clinical Manager, Cancer Assessment Clinic and Women's Breast Health Centre, The Ottawa Hospital, 501 Smyth Rd., Ottawa, ON K1H 8L6. Email: jesmylie@TOH.on.ca
Ontario. The creation of this model led to the development of The Ottawa Hospital (TOH) Cancer Assessment Clinic (CAC) that serves as the gateway for access to cancer services for the region. Clinical and administrative leadership working towards a common goal of streamlining prostate cancer care resulted in the opening of the Prostate CAC in May 2008. Supporting the multidisciplinary work of the CAC is another component of the regional model: the Champlain Prostate Cancer Community of Practice (CoP). This network brings together health care professionals from across the region involved in the care of prostate cancer patients. In addition, it provides a platform for relationship building, knowledge exchange, gap analysis, development of quality initiatives and quality indicator data collection (Fung-Kee-Fung, Watters, Crossley, et al., 2009). The Prostate CAC team provides leadership in the implementation and evaluation of these CoP quality initiatives.

The Prostate CAC ensures coordinated quality patient care from referral to definitive diagnosis (Brouwers et al., 2007). The objectives of the Prostate CAC are to increase access to evidence-based inter-professional care, reduce wait times for diagnosis, reduce wait times for treatment through coordination of care, and provide support and counselling to address the psychosocial needs of patients. The inter-professional team working in the Prostate CAC includes urologists, radiologists, radiation oncologists, social workers, ultrasound technologists, and nurses.

\section{Process redesign}

Prior to the opening of the Prostate CAC, there was impetus for change. Concerns included prolonged transrectal ultrasound-guided (TRUS) biopsy wait times, which delayed diagnosis and treatment planning causing undue psychological distress for patients. In addition, services were located at different campuses and managed by various departments, often resulting in reduced communication and fragmentation of care. Clinicians and administrators began working together to plan for improvement. Following a review of the current state, the focus was on process redesign, aligning demand and capacity, and mapping the new trajectory of care for assessment and diagnosis. The leadership team collaborated to set wait time targets that were embedded within the new process map.

In conjunction with the process improvement initiatives, there was the intent to provide patient-centred care and improve the patient experience. It was recognized that patients face many challenges during the diagnostic phase of care, including communication with and between health care providers (Cancer Quality Council of Ontario, 2007). Strategies were introduced to begin to address these issues. When the Prostate CAC opened, a streamlined referral process supported centralized access, TRUS biopsy activity was located within the clinic, and an inter-professional team was created to enhance the quality and safety of care provided to patients (Canadian Medical Association, 2008).

\section{Improving the patient experience}

From the inception of the Prostate CAC, nurses have played an essential role in the development of new initiatives to positively impact the patient experience. The importance of communication is noted in all of the nurse-led initiatives, including the information guide and personal record, TRUS biopsy care, patient resources, community links, the implementation of a decision aid for early stage prostate cancer, and surgery education classes. 
Information guide and personal record

One initial strategy to improve communication and provide information is the Prostate Cancer Information Guide and Personal Record, given to every patient at the CAC at the time of diagnosis. It is modelled after a similar guide in use at TOH's Women's Breast Health Centre (WBHC), and highly valued by patients. Many health care professionals participated in the development of the guide but, more importantly, patients were integral to the design, content, and layout of the guide. This ensured that the end product was patientfocused, functional and addressed patient needs. The guide empowers the patient to:

- Track appointments/schedules/test results,

- Create a personal diary; record their own information and any questions,

- Understand information on prostate cancer including treatment options and side effects,

- Gain access to resources, community supports and medical terminology.

The guide is provided for use from diagnosis through treatment and onwards, serving as a communication tool between the patient and the inter-professional team.

\section{Transrectal ultrasound-guided prostate biopsy care}

Transrectal ultrasound-guided prostate (TRUS) biopsy is a highly invasive, occasionally painful, and sometimes traumatic event that is surrounded by heightened fear of the diagnosis of a malignant and life-threatening disease (Wareing, 2004). Risks associated with TRUS biopsy include bleeding, infection and urinary retention (El-Hakim \& Moussa, 2010; Lindert, Kabalin \& Terris, 2000; Saad \& McCormack, 2008; Wareing, 2004). Hospital admissions following TRUS biopsies have increased over the past 10 years, primarily attributed to a growing rate of infection-related complications (Nam, Saskin, Lee, et al., 2010).

Another early goal of the Prostate CAC was to coordinate care to improve wait times, safety, patient education and the overall experience of men undergoing a TRUS biopsy. Historically, the wait time from request to TRUS biopsy varied between eight and 12 weeks. Prior to the Prostate CAC opening, key individuals met to address TRUS biopsy wait times and have continued to meet regularly to attend to issues and concerns as they arose. Communication and collaboration with radiologists and ultrasound administration have resulted in designated biopsy spots for Prostate CAC patients in the booking schedule.

Today, with a responsive system in place to monitor wait times and align demand and capacity, delays in scheduling lead to prompt requests for additional biopsy spots, as needed. From opening to present day, the wait time target of 14 days or less from request to TRUS biopsy completion has been sustained (see Figure 1). In addition, a patient-centred approach has ensured that everyone leaves their consult visit with a biopsy appointment, a written plan of care and a follow-up date for results. Decreasing the wait time from consults to biopsy, and then to a follow-up for results discussion with an urologist has significantly improved the patient experience.

Shortly after the opening of the Prostate CAC, the identification of bacteremia in patients following TRUS biopsy prompted a thorough review of the existing antibiotic prophylaxis, reprocessing systems, environmental cleaning, and procedural and hand hygiene practices. Following a literature review, new guidelines for antibiotic prophylaxis were developed with leadership from urologists, infectious disease physicians and the pharmacy team. The physicians and nurses in the Prostate CAC were instrumental in communicating these new guidelines to health care providers across the region.

Following the new guidelines, patients meeting the criteria for higher risk of bacteremia receive an intramuscular injection of antibiotics one hour prior to the TRUS biopsy procedure, given by a nurse in the CAC. Similarly, prior to the opening of the Prostate CAC, patients who had not taken their appropriate oral antibiotics were routinely cancelled and rescheduled. Today, rather than cancelling their procedure, these patients receive an injection from the nurse, thus avoiding patient delay and frustration, and improving efficiency to the overall health care system.

With the ultrasound procedural rooms on-site in the CAC, nurses are now available to respond to biopsy patient emergencies such as patient vasovagal episodes, chest pain and cardiac arrest. Each procedure room is equipped with an emergency response system and a nurse is routinely assigned for TRUS biopsy patient coverage. Radiologists and sonographers have voiced their appreciation of the team approach:

I wanted to let you know how impressed the radiologists and sonographers are with your team and the support you have provided during vasovagal episodes. The quick response from your nursing staff is truly remarkable and much appreciated.... Thank you!

-Ultrasound Administration, 2010

Research has shown that preparing a patient for a procedure with both factual and sensory information will decrease anxiety and increase satisfaction (Langhorne, Fulton \& Otto, 2007). The Prostate CAC team has optimized the preparation of patients undergoing a TRUS biopsy with consistent education provided by nurses. Patients receive a detailed preparation sheet for pre and post biopsy care explaining the nature, risks and possible side effects, as well as specific instructions regarding anticoagulation and antibiotic prophylaxis. Contact information is also provided. Many patients and their families appreciate that they can speak directly to a nurse and have questions or concerns promptly addressed.

An important initiative was recently implemented to help improve emergency care for patients post TRUS biopsy. Nursing, in collaboration with urology, developed emergency visit instructions

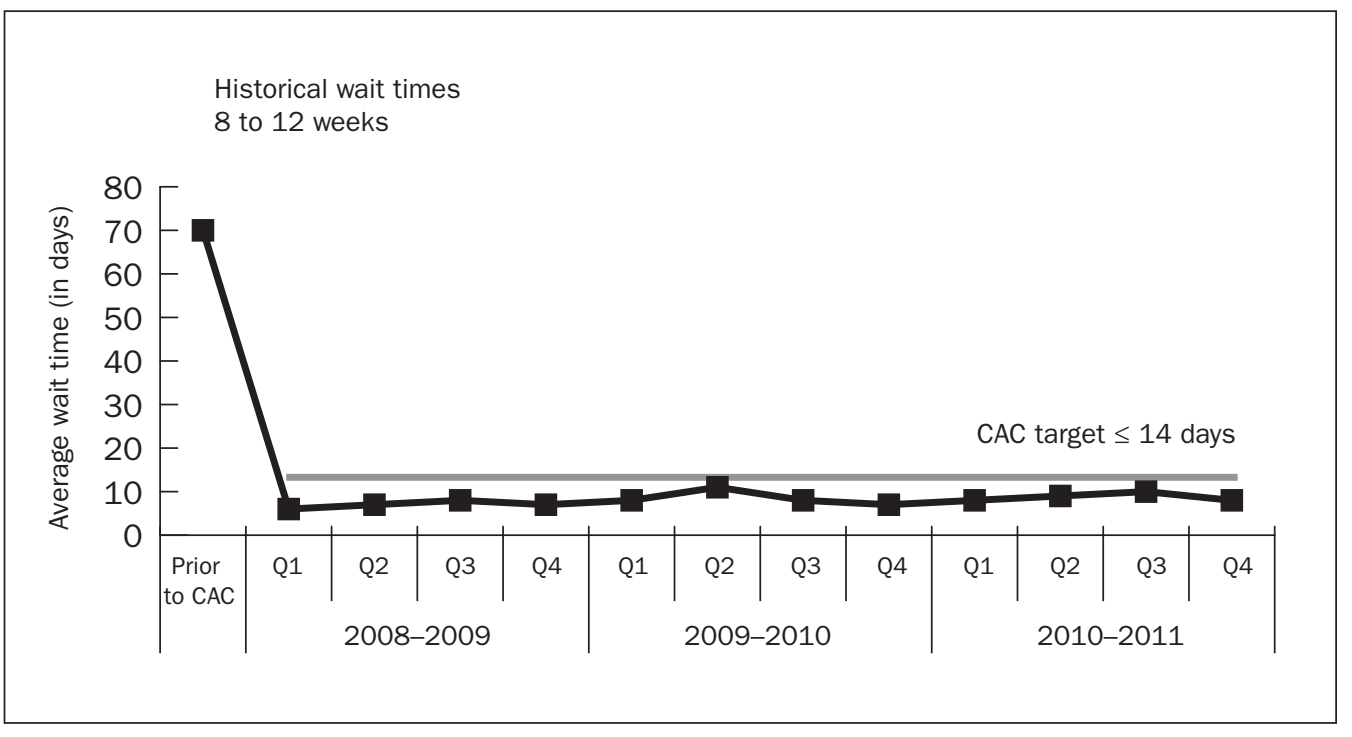

Figure 1: Prostate CAC TRUS biopsy wait times 
for patients should they experience fever and/or chills post biopsy. Patients are instructed to keep the emergency visit instructions in their wallet following their biopsy, and advised to present these instructions to the hospital emergency department staff upon arrival. Signs and symptoms of infection or sepsis could be due to an increased resistance to standard antibiotic treatment. Patients and families are informed that bacteremia can develop very quickly post biopsy and put the patient at risk of critical illness and death (Lindert, Kabalin \& Terris, 2000). These instructions serve as a communication tool between the patient and health care providers, ensuring that the patient receives appropriate care in a timely fashion.

TRUS biopsy procedural pain is largely unrecognized or overlooked, and men have reported varying levels of pain (Oliffe, 2004). Pain is a subjective experience and the patient's self report of pain is the single most reliable indicator of pain (McCaffery \& Pasero, 1999). TRUS biopsy pain can be influenced by psychosocial factors such as anticipated physical discomfort, issues of individual mortality, cultural inappropriateness of the procedure itself and anal penetration causing embarrassment (Oliffe, 2004). With the Prostate CAC serving as a central access point for TRUS biopsy, it was soon recognized by both physicians and nurses that patients had concerns with the level of pain they experienced during their biopsy procedure. As one patient stated:

I was able to manage the pain during the first few bites, but by

the time they got to the fifth and sixth bites it was unbearable.

-TRUS biopsy patient, 2008

In response to patient pain concerns, in early 2009, the Prostate CAC nurses developed and implemented a patient quality improvement initiative to survey patients regarding the level of pain they experienced during TRUS biopsy. Patients reported the level of pain they experienced and whether they had received "freezing" for the procedure. Results clearly demonstrated that patients who received a periprostatic nerve block experienced significantly lower levels of pain (see Figure 2). A summary of the survey was shared in a prostate team meeting with radiologists, urologists and nurses. It has since become standard practice at $\mathrm{TOH}$ to give every TRUS biopsy patient a periprostatic nerve block. The use of anesthesia for TRUS biopsy has also been written into our regional guidelines. These recommendations are also found in the Canadian Urological Association guidelines (El-Hakim \& Moussa, 2010). The use of a periprostatic nerve block for TRUS biopsy has significantly decreased patient anxiety and pain. As stated by a TRUS biopsy patient:

This was my fourth biopsy and I was very anxious about the pain. I received freezing this time and it was a breeze compared to the other times without.

-TRUS biopsy patient, 2009

Nurses have made a significant contribution to improving the overall patient experience for TRUS biopsy with reduced wait times, standardization of antibiotic prophylaxis and radiology practices, as well as improvements in pre and post teaching. As one patient praised:

Your caring attitudes made a scary time less anxiety-filled. Thank you for your professionalism and kindness before, during and after my biopsy.

-TRUS biopsy patient, 2009

\section{Patient resources}

Upon diagnosis of prostate cancer, patients and their families have access to the support and guidance of the interprofessional team members in the Prostate CAC. In addition, the team has focused on providing written material, allowing patients and families to review information at their own pace. Nurses emphasize that the information is there for them when they are ready, always aware that patients and their families may be overwhelmed with the amount of information they receive. At present, resources given to newly diagnosed men along with the Prostate Cancer Patient Information Guide and Personal Record include:

- Understanding Prostate Cancer (Saad \& McCormack, 2008)

- A patient resource handout that includes social work contact information, community and financial resources and community support groups

- A decision aid for men who are diagnosed with an early stage prostate cancer.

Many patient education books, pamphlets, and publications are housed within the CAC Patient Resource Centre.

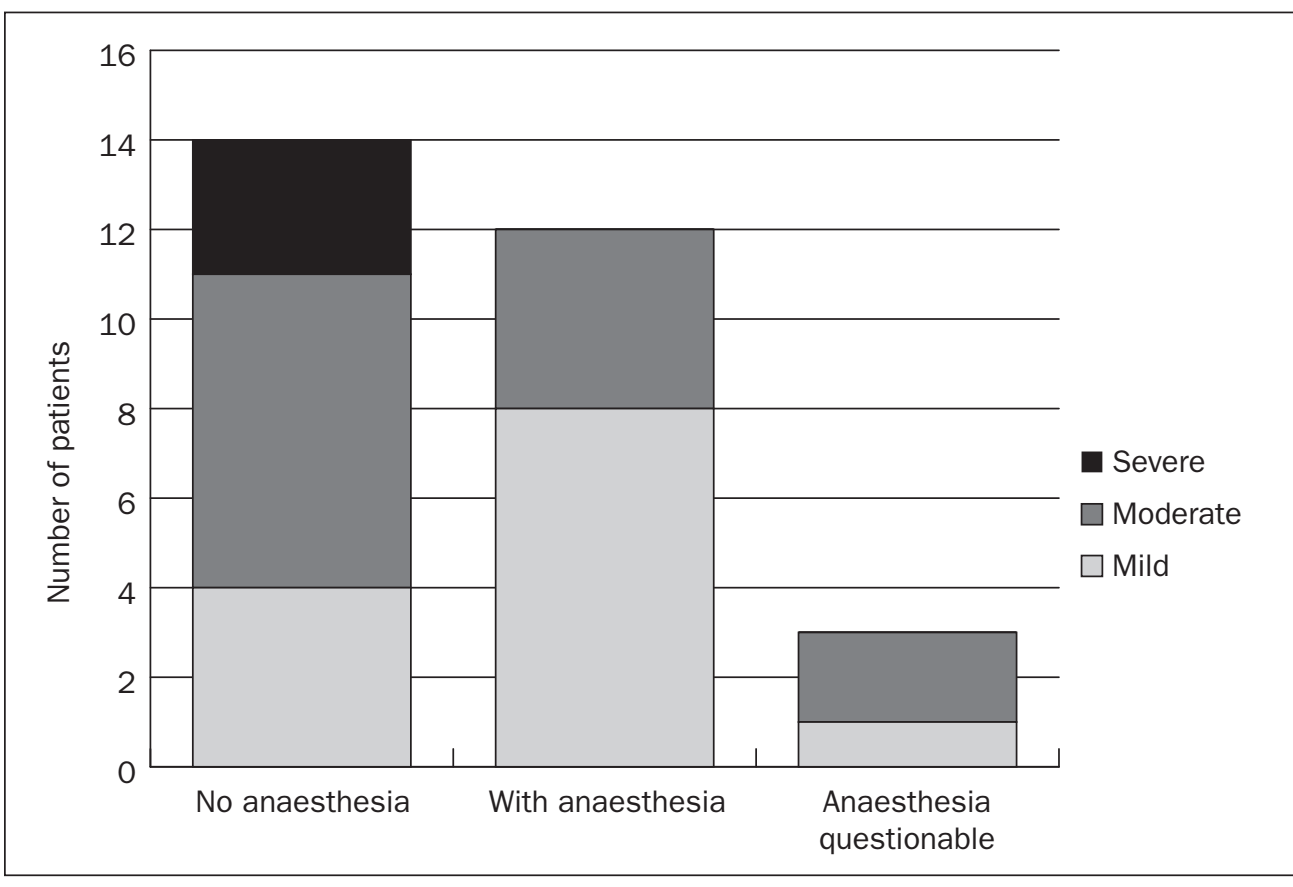

Figure 2: Patient Quality Improvement survey results n=29, March/April 2009

\section{Community links}

Health and illness information are integral to the well-being of men diagnosed with prostate cancer. Support groups have emerged as important communitybased resources with studies indicating that men derive a sense of meaning and purpose through attending support group meetings (Oliffe, Bottorff, Hislop, \& McKenzie, 2009). The Prostate Cancer Canada Network (PCCN) Ottawa is part of a national support group for men living with prostate cancer. The Prostate CAC has developed a close communication link with the Ottawa Chapter and, on several occasions, urologists, nurses, administrators and social workers have attended meetings and presented new initiatives for feedback to the group. The Walnut, a newsletter of the PCCN Ottawa, keeps prostate cancer patients and their families in touch with recent events and current research. All newly diagnosed men are given contact information for the Ottawa support group, and many have attended their mentoring sessions and meetings. 
Nurses in the Prostate CAC have linked with another support group reaching out specifically to gay men in Ottawa. It is estimated that 1,500 gay and bisexual men in Canada develop prostate cancer each year (Ryerson University - Psychosocial Medicine Lab, n.d.). Gay men are likely to be affected differently by prostate cancer in terms of understanding masculinity, sexuality, social relationships and relationships in the health care community (Asencio, Blank, Descartes, \& Crawford, 2009; Blank, 2005; Fergus, Gray \& Fitch, 2002; Gray 2002; Perlman \& Drescher, 2005). The founder of Gay Buddies with Prostate Cancer described that it would have been easier for both him and his partner to face the diagnosis of prostate cancer if he could have spoken with other gay men who had survived prostate cancer and learned to live with the challenges (McKinnon, 2006).

Many gay men don't feel safe or comfortable discussing their sexual concerns in a straight-focused prostate cancer support group (Blank, 2005; Filiault, Drummond, \& Smith, 2008; Perlman \& Drescher, 2005; Santillo, 2005). Our central access system allows us to direct patients to the appropriate supports and resources in our community. Providing an atmosphere of inclusivity in our clinic has opened the door to more meaningful communication with gay men and their partners. Prostate CAC nurses were invited to the first Ottawa showing of the film A Dance with Cancer-5 Gay Men Confront Prostate Cancer (Canadian Cancer Society, 2010). The film explores the personal stories and real life experiences of five gay men who are dealing with prostate cancer. The Canadian Cancer Society now sponsors the initiative and has provided copies that are given to patients in the CAC. An example of nursing leadership was to connect the Gay Buddies with Prostate Cancer support group with the Canadian Cancer Society, linking the informal with the formal.

\section{Implementation of a decision aid}

Decision-making can be an agonizing experience for men diagnosed with an early stage prostate cancer. The side effects and complications vary across treatment options, namely urinary incontinence, erectile dysfunction and bowel concerns, and many men are unsure about what treatment is best for them. The importance of shared decision-making is clear where patients have a choice between treatment modalities that offer similar survival outcomes, such as surgery versus radiotherapy for early stage prostate cancer (Eggertson, 2010). Decision aids are designed to complement counselling from health care practitioners, assisting the patient to play an active role in decisions regarding their health (Stacey, Legare, \& Kryworuchko, 2009).

An important role in nursing is to provide education to patients, and decision aids help nurses provide this education and support for patients making difficult decisions (Eggertson, 2010). Initial discussions regarding the use of a decision aid for men diagnosed with early stage prostate cancer began in 2007. The research team included nurses, information technology specialists, radiation oncologists and urologists. Focus groups and interviews were conducted with many stakeholders. Following a lengthy process, a decision aid was selected. It consists of a DVD and an accompanying booklet giving up-to-date information about prostate cancer, including the associated benefits and harms of the different treatment choices.

The use of the decision aid was initiated in September 2010. Appropriate patients are loaned a decision aid at diagnosis, with sufficient time to take home for review. In addition, patients are asked to complete the Personal Decision Form, a short questionnaire to assess knowledge, values, preferences, and where the patient is in the decision-making process. When the booklet, DVD and questionnaire are returned, nurses in the Prostate CAC are available to provide decision support, additional resources and referrals, if required. Urologists and radiation oncologists in the Prostate CAC receive a one-page summary of the patient's clinical and decisional data from the completed Personal Decision Form questionnaire. This information is used to communicate the patient's preferences in the patient/ physician encounter, a clear example of shared decision-making.

To date, more than 100 decision aids have been given to men diagnosed with early stage prostate cancer. Nursing is the critical link for getting the decision aid into the patient's hands (Eggertson, 2010). This is the first known decision aid initiative in Canada to be implemented for sustained use within a clinical setting and has led to interviews of key nursing members of the research team (Eggertson, 2010). In addition, interested teams from other regions have made site visits to the Prostate CAC. CAC nurses recently participated in a panel presentation at the Inter-professional Shared Decision Making Workshop held at the University of Ottawa, March 2011. Most importantly, comments from our patients have been very positive:

I found it very informative and the treatment options were very well explained. It helped to consolidate all the information I had and make it more meaningful. Thank you.

My wife and I were at a loss as to what was the best option for me. The DVD and booklet really helped us to make a decision and feel confident about it.

-CAC prostate cancer patients 2011

\section{Surgery education classes}

It is well documented in the literature that the time from diagnosis to treatment is fraught with anxiety for patients and their families (Langhorne, Fulton, \& Otto, 2007). Surgery education classes for patients undergoing a radical prostatectomy commenced in September 2009. Information discussed in the class includes diet, exercise, pre-operative preparation, what to expect during the hospital stay, post-operative recovery, resources and support. In addition, the teaching session focuses on catheter care, managing

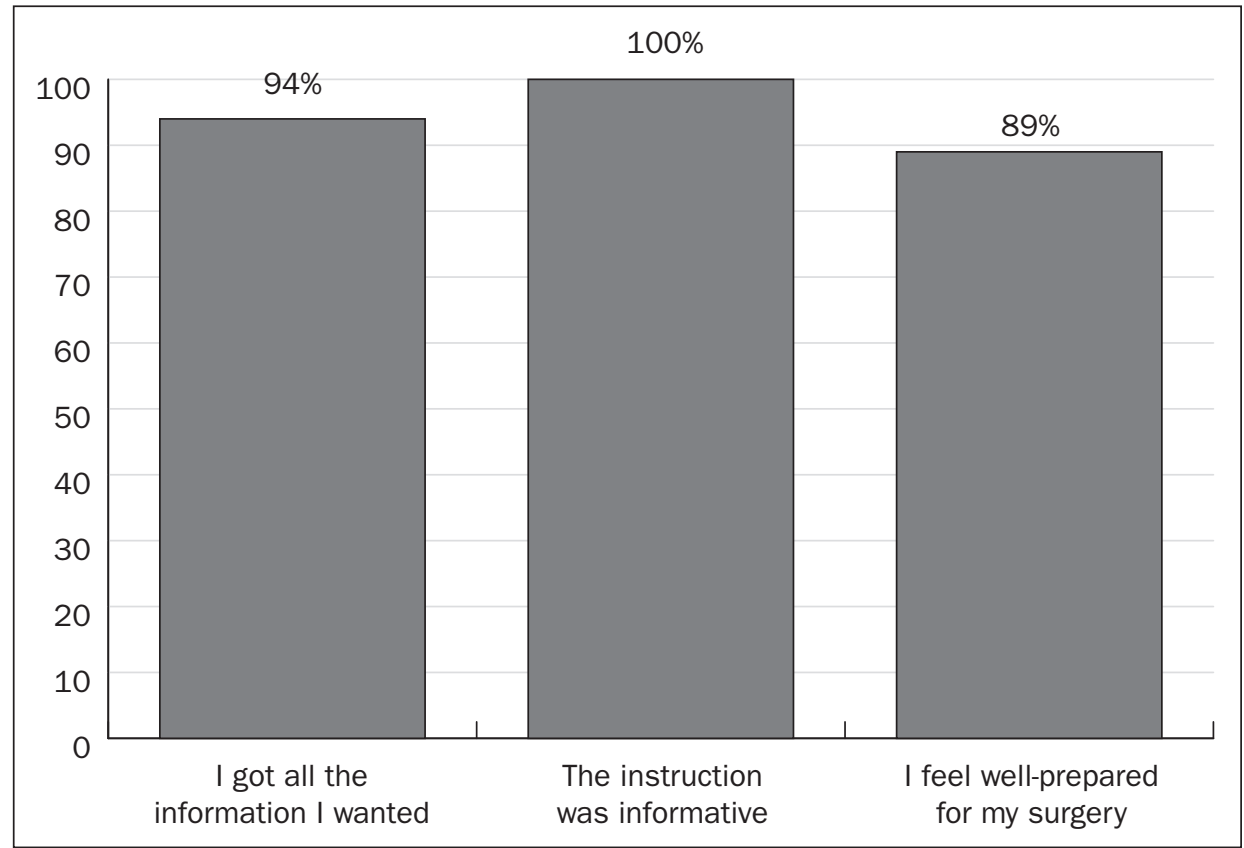

Figure 3: CAC prostate questionnaire

n=35, January 1, 2011-March 31, 2011 
incontinence and erectile function concerns post surgery. It is extremely important that men and their families receive education and information prior to their treatment about urinary and sexual difficulties post treatment and what can be done about it (Mulhall, 2008). The class is nurse-led, with social work playing an important role in providing information regarding psychosocial oncology supports and resources. Evaluations of the teaching sessions have been very positive (see Figure 3).

\section{Future initiatives}

The inter-professional team approach, expertise, leadership and the non-institutional setting of the Prostate CAC are integral in improving the patient experience for men newly diagnosed with prostate cancer. A future initiative for the nursing team is the development of self-directed learning modules for nurses, to promote knowledge translation and best practices in regards to prostate cancer. There has also been increasing interest in sharing nursing expertise and practices with both regional community partners, and across the province.

\section{REFERENCES}

Asencio, M., Blank, T., Descartes, L., \& Crawford, A. (2009). The prospect of prostate cancer: A challenge for gay men's sexualities as they age. Journal of NSRC, 6(4), 38-41.

Blank, T.O. (2005). Gay men and prostate cancer: Invisible diversity. Journal of Clinical Oncology, 23, 2593-2596.

Brouwers, M., Crawford, J., Elison, P., Evans, W., Gagliardi, A., Holmes, D., et al. (2007). Organizational standards for diagnostic assessment programs: Recommendations. Retrieved from http://www.cancercare.on.ca/common/pages/UserFile. aspx?fileId $=36870$

Canadian Cancer Society (2010). A dance with cancer-5 gay men confront prostate cancer. Retrieved from http://www.cancer.ca/ Ontario/Support\%20Services/Videos/A\%20dance\%20with\%20Can cer\%20-\%205\%20Gay\%20Men\%20Confront\%20Prostate\%20Cancer. aspx?sc_lang=en

Canadian Cancer Society (2011). Prostate cancer statistics at a glance. Retrieved from http://www.cancer.ca/Ontario/About\%20cancer/ Cancer\%20statistics/Stats\%20at\%20a\%20glanceProstate\%20 cancer.aspx?sc_lang=en

Canadian Medical Association Policy (2008). Achieving patientcentred collaborative care. Retrieved from http://policybase.cma. ca/dbtw-wpd/Policypdf/PD08-02.pdf

Cancer Quality Council of Ontario (2007). "Is it cancer? Improving the patient Journey to Diagnosis" The patient experience from symptoms to diagnosis. Retrieved from http://www.cqco.ca/cms/ One.aspx?portalId $=89613 \&$ pageId $=89747$

Cayne, B., Lechner, D., et al. (1988). Webster's Encyclopedic Dictionary of the English Language (Canadian edition). New York: Lexicon Publications, Inc.

Eggertson, L. (2010). Patient decision aids help answer "What's best for me". Canadian Nurse, 106(9), 38-42.

El-Hakim, A., \& Moussa, S. (2010). CUA guidelines on prostate biopsy methodology. Canadian Urological Association, 4(2), 89-94.

Fergus, K.D., Gray, R.E., \& Fitch, M.I. (2002). Sexual dysfunction and the preservation of manhood: Experiences of men with prostate cancer. Journal of Health Psychology, 7(3), 303-316.

Filiault, S.M., Drummond, J.N., \& Smith, J.A. (2008). Gay men and prostate cancer: Voicing the concerns of a hidden population. Journal Men's Health, 5(4), 327-332.

Fung-Kee-Fung, M., Watters, J., Crossley, C., Goubanova, E., Abdulla, A., Stern, H., \& Oliver, T. (2009). Regional collaborations as a tool for quality improvements in surgery: A systematic review of the literature. Annals of Surgery, 249(4), 565-572.
Overall, nurses have played a significant role in increasing the coordination of care, decreasing wait times from assessment to diagnosis, providing education, guidance and decision support to patients diagnosed with prostate cancer. The impact on patients and their families are reflected in their comments:

Thank you. It was a difficult time for me. Your caring, kindness and support were very much appreciated.

Your help during my cancer treatment made it much easier to deal with. Your caring attitudes made a scary time less anxietyfilled.

-CAC prostate cancer patients 2011

Communication is the key to improving the prostate cancer patient and family experience.

\section{Acknowledgement}

We would like to thank Heather Lloyd-Easy, RN, BSCN, CON(C), for her contributions in the Prostate Cancer Assessment Clinic.

Gray, R. (2002). Prostate tales-men's experiences with prostate cancer. Harriman, TN: Men's Studies Press.

Langhorne, M., Fulton, J., \& Otto, S. (2007). Oncology Nursing (fifth edition). St. Louis, MO: Mosby Elsevier.

Lindert, K., Kabalin, J., \& Terris, M. (2000). Bacteremia and bacteriuria after transrectal ultrasound guided prostate biopsy. Journal of Urology, 164(1), 76-80.

McCaffery, M., \& Pasero, C. (1999). Pain Clinical Manual (second edition). St. Louis, MO: Mosby, Inc.

McKinnon, N. (2006, February 23). Gay buddies with prostate cancer. Retrieved from http://www.xtra.ca/public/printStory.aspx? AFF_TYPE=2\&STORY_ID=1399

Mulhall, P. (2008). Saving Your Sex Life-A guide for men with prostate cancer (first edition), Chicago, IL: Hilton Publishing Company.

Nam, R., Saskin, R., Lee, Y., Liu, Y., Law, C., Klotz, L., et al. (2010). Increasing hospital admission rates for urological complications after transrectal ultrasound guided prostate biopsy. Journal of Urology, 183(3), 963-8.

Oliffe, J. (2004). Transrectal ultrasound prostate biopsy (TRUS-Bx): Patient perspectives. Urologic Nursing, 24(5), 395-400.

Oliffe, J., Bottorff, J., Hislop, T.G., \& McKenzie, M. (2009). The role of prostate cancer support groups (PCSGs) in health promotion: Executive summary. Retrieved from http://www. menshealthresearch.ubc.ca/Links.aspx

Perlman, G., \& Drescher, J. (2005). A Gay Man's Guide to Prostate Cancer. Binghamton, MA: Haworth Press.

Ryerson University - Psychosocial Medicine Lab. (n.d.). Quality of life among gay/bisexual men with prostate cancer study and their partners. Retrieved from http://www.ryerson.ca/psychmed/ researchprojects/gmpca/index.html

Saad, F., \& McCormack, M. (2008). Understanding prostate cancer. Montreal: Rogers Business and Professional Publishing Group.

Santillo, V.M. (2005). Prostate cancer and the gay male. Journal Gay \& Lesbian Psychotherapy, 9(1/2), 155-170.

Stacey, D., Legare, F., \& Kryworuchko, J. (2009). Evidence-based healthcare decision making: Roles for health professionals. In A. Edwards \& G. Elwyn (Eds.), Evidence-based shared decision making in healthcare: Achieving evidence based patient choice (2nd ed.). Oxford: Oxford University Press.

Wareing, M. (2004). Transrectal ultrasound and biopsy clinic. Nursing Standard, 18(24), 33-37. 